\title{
Face adaptation: Changing stable representations of familiar faces within minutes?
}

\author{
Claus-Christian Carbon ${ }^{1,2}$ \& Helmut Leder $^{1}$ \\ ${ }^{1}$ Department of Psychological Basic Research, University of Vienna, Austria \\ ${ }^{2}$ Institute of Cognitive and Biological Psychology, Freie Universität Berlin, Germany
}

Received 07 April, 2005

Accepted 01 June, 2005

\section{Keywords}

face recognition, face representation, adaptation effect, learning, memory

\begin{abstract}
Three experiments are reported showing that the perception and the assessment of veridicality of familiar faces are highly adaptive to new visual information. Subjects were asked to discriminate between real photographs and altered versions of celebrities. Exposing participants to extremely deviated versions changed the usually stable representations of the famous faces within a very short time. In Experiment 1, expo-
\end{abstract}

sure to an extreme face version resulted in identity decisions shifted towards the exposed one. Experiment 2 revealed that the effects are not short lasting. In Experiment 3, we showed that the effect also generalizes to different pictures of the same famous person. Together the experiments seem to indicate that the brain permanently adapts to new perceptual information and integrates new data within already elaborated representations in a fast way.

\section{INTRODUCTION}

In order to recognise a previously seen face very quickly (Carbon \& Leder, in press) and accurately, it is commonly thought that incoming perceptual information must be matched against representations of faces stored in memory (Bruce \& Young, 1986). Furthermore, theories of memory implicitly claim that these stored representations are both stable and accurate, containing the essential information that allows a face to be recognised (Bruce, 1994; Leder \& Carbon, 2005). However, here we show that representations of faces in memory are rather more flexible, being subject to immediate adaptation by exposure to new visual information.

Human long-term memory holds information about objects, events and affective evaluations (Bower, Thompson-Schill, \& Tulving, 1994). Representations of objects serve their recognition, and are a prerequisite for selecting adequate actions (Baddeley, 1998). In order to recognise objects, which might be encountered from different viewpoints, with different light- ing and context conditions (Leder \& Carbon, 2005), researchers have suggested that these representations comprise essential information about an object that is invariant across such changes. Faces present a particular problem for recognition, as, in addition to changes in viewpoint and lighting, faces appear different from instant to instant because of changes in expression, hairstyle, age and speech-related movements (Lander \& Chuang, in press). Once again, the solution to this problem has been to assume that invariant structural information is abstracted from faces, which allows their recognition despite such changes. These representations are thought to be based on the component features of the face as well as their configuration (Leder \& Bruce, 2000; Leder \& Carbon, 2004, in press). Moreover, a number of theories claim that face recognition proceeds by using prototype

Correspondence concerning this article should be addressed to Claus-Christian Carbon, who is now at the Department of Psychological Basic Research, University of Vienna, Liebiggasse 5, A-1010 Vienna, Austria. Electronic mail may be sent via Internet to ccc@experimental-psychology.com 


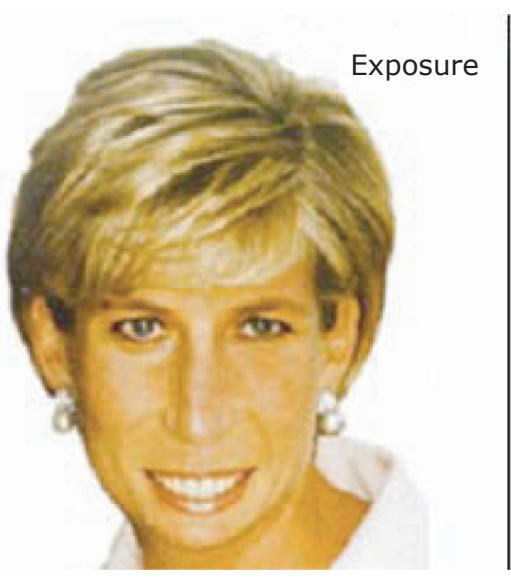

$1 a$

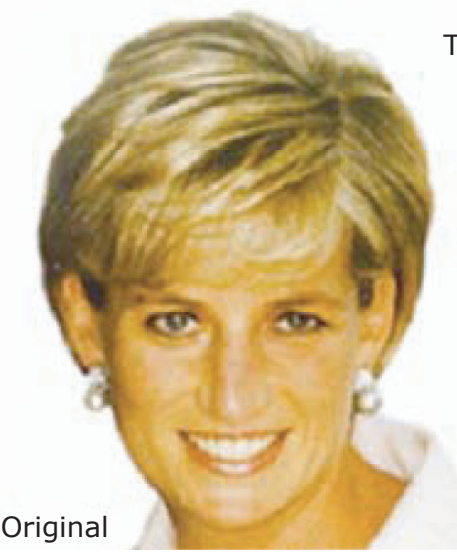

$1 \mathrm{~b}$

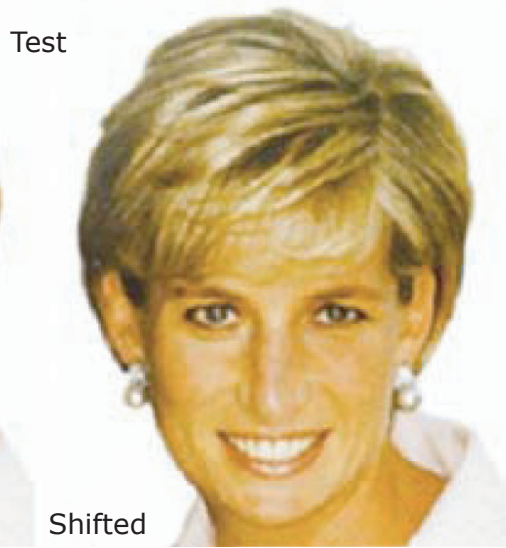

$1 \mathrm{c}$

\section{Figure 1.}

Princess Diana as one of the celebrities used as stimulus material in Experiment 1. On the left the EXTREME, in the middle the original version (ORIGINAL), and at the right shifted version (SHIFTED). The question in the test phase was "Is this picture the veridical image of Princess Diana?".

(Benson \& Perrett, 1993) processing, which is related to a represented ideal exemplar of a face (Rhodes, Jeffery, Watson, Clifford, \& Nakayama, 2003).

However, rather than a relatively stable representation of a face, an alternative suggestion is that a more flexible adaptation mechanism exists, which updates the representation in memory when the visual experience reveals a change in appearance. For example, recognizing familiar faces despite short or long-term changes due to hairstyles is probably most efficiently done by integrating a new hairstyle into the representation of that person's face. Here we ask whether this kind of adaptation is found for the more fundamental aspects of face representations such as the configuration of face features. We did so by first exposing people to versions of highly familiar faces in which the distance between face features was extremely exaggerated (EXTREMEs). We then examined the effects of this exposure on judgements of veridical and less exaggerated versions of the same faces. If the memory representations adapt immediately, we expect participants to judge falsely a slightly exaggerated version of the face as veridical, and be less certain about the veridicality of a veridical version. However, if the underlying representation is unaffected by apparently exaggerated information - perhaps because memory representations reflect the amount of exposure frequency - then the judgements about veridical representations should remain unchanged. It is important to point out that the EXTREME versions are not caricatures in which distinctive features are systematically exaggerated and sometimes reveal better recognisable representations. Instead, the al- terations used in our experiments were uncorrelated with the distinctive dimensions of any face features. Furthermore, we used highly familiar faces in contrast to most studies investigating the figural aftereffect with unfamiliar faces (e.g. Webster \& MacLin, 1999).

\section{THE PRESENT STUDY}

The aim of the present study was to analyze the adaptation effect found by other researchers (e.g., Webster \& MacLin, 1999) when using familiar faces, here realized by presenting celebrities (Experiment 1). Moreover, we wanted to investigate whether the adaptation effect is a perceptual, short termed effect or a longer lasting effect, supposedly based on a memory-based mechanism (Experiment 2). Furthermore, we were interested whether the effect is a pictorial or a structural one (Experiment 3), or whether it is an unspecific alteration of (face) perception in a more general sense.

\section{EXPERIMENT 1}

This experiment investigated whether one shortly presented distorted face version in the exposure phase reduces the ability to discriminate veridical and slightly altered faces. In order to avoid interpretations due to simple visual afterimages, we used visual backward masking following the exposure.

\section{Method}

\section{Subjects}

Twenty undergraduate participants from the Freie Universität Berlin were tested individually (16 females, mean age 27.0 years). Participants were either paid 

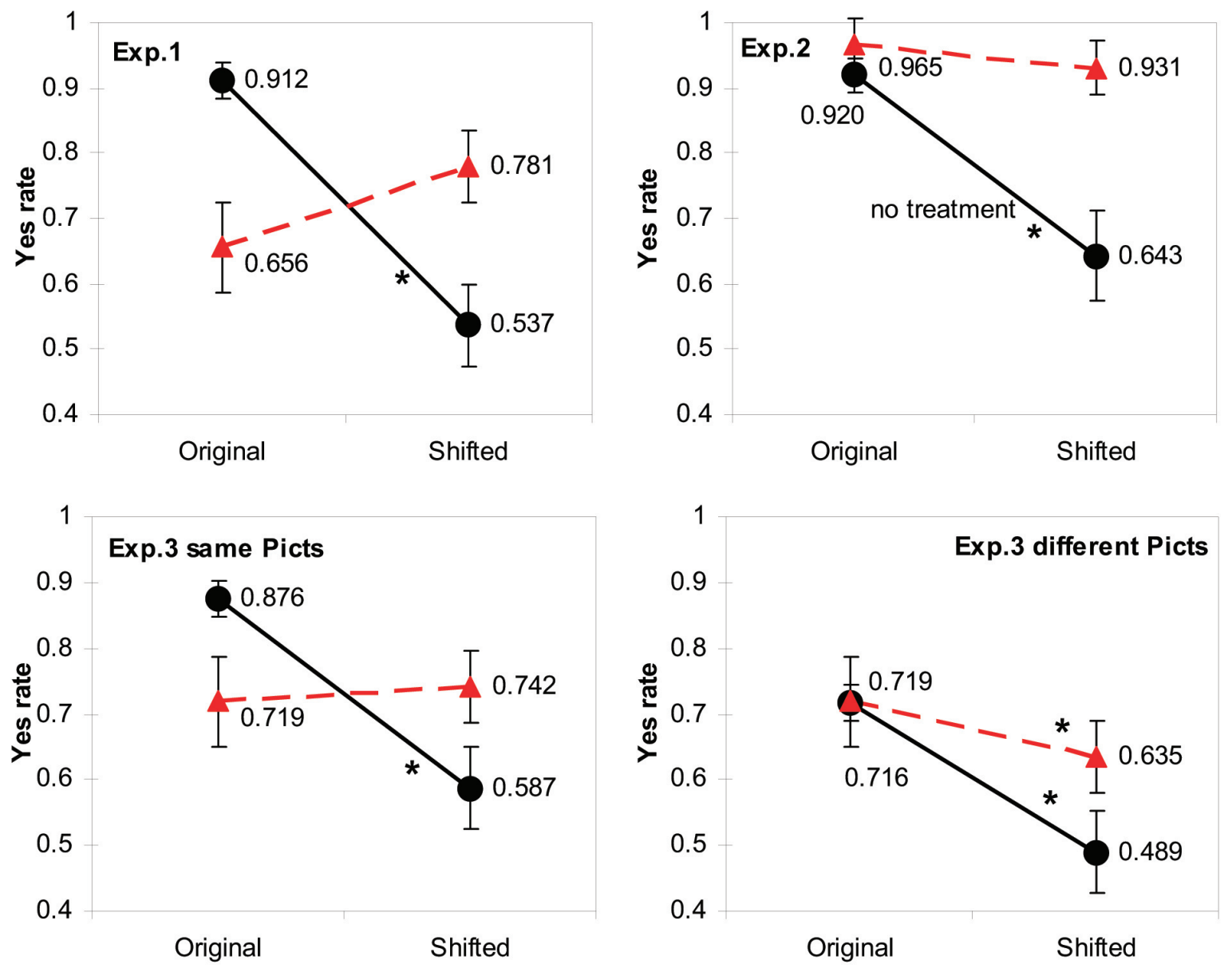

\section{Figure 2.}

Averaged "Yes-rate" data for Experiment 1, 2, and 3. Error bars are SEs; asterisks indicate significant differences between conditions.

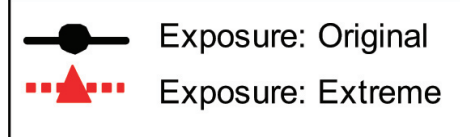

or recruited as part of study requirements. All participants in the following experiments had normal or corrected-to-normal vision. They were naïve to the purpose of the experiment and each participant took part only once in the experiments reported here.

\section{Apparatus and stimuli}

Color photographs of eight well-known celebrities were used (Princess Diana, C. Diaz, J. Roberts, C. Crawford, G. Depardieu, B. Pitt, P. Brosnan, G. Clooney). Each stimulus face was used in three different versions. The ORIGINAL version was a veridical version of a familiar picture of the celebrity (see Figure 1b). Additionally, two increased versions were created based on this ORIGINAL version. In the EXTREME version, the distance between the eyes and the mouth was extremely increased, while the position of the nose remained unchanged (see Figure 1a). This shifting is similar to other studies using configurally distorted unfamiliar facial material (Faulkner, Rhodes, Palermo, Pellicano, \& Ferguson, 2002). In the SHIFTED version, the distance between the eyes and nose regions was only slightly increased (see Figure 1c). However, both altered versions (EXTREME and SHIFTED) could be identified as belonging to the same celebrity. The size of the pictures was about $220 \times 240$ pixels.

\section{Procedure}

At the beginning of the experiment, participants had to name the faces of the celebrities and gave information about their profession. During the exposure, the participants inspected an ORIGINAL or an EXTREME version of a celebrity including her/his name for 30 seconds. This was followed by a random dot mask (duration: $3000 \mathrm{~ms}$ ) and a blank field (duration: $1000 \mathrm{~ms}$ ) to erase possible afterimages (Turvey, 1973) from iconic memory (Coltheart, 1980; Neisser, 1967). After this masking procedure, either an ORIGINAL or a SHIFTED version of a test face appeared on the screen. Participants decided in a Yes-No decision task whether or not the test stimulus was a veridical version of the celebrity's face known from television, movies or the 
press. They were asked to ignore the preceding exposed face and to base their decision completely on their knowledge about the celebrities from the media. Thus, it was emphasized that they should judge whether each test stimulus was an accurate representation of the face as encountered on television, in the cinema or in the press. After the participants had made their choice the next trial started automatically. The trials, presented in a randomized order, were controlled by the experimental control software PsyScope PPC 1.25 (Cohen, MacWhinney, Flatt, \& Provost, 1993), running on a Macintosh Imac with an integrated 15-inch CRT (1024 x 768 pixels). The design was fully balanced, i.e. each of the possible $2 \times 8$ exposure faces (EXTREME vs. ORIGINAL) was crossed with every possible T-face (ORIGINAL vs. SHIFTED), resulting in 32 total trials.

\section{Results and Discussion}

In all experiments reported here only faces that were familiar to each participant, were further analyzed. The rate of familiarity was in no experiment less than $90 \%$.

Participants were very accurate in distinguishing between the ORIGINAL and the SHIFTED version, after having been exposed to an original version. This was measured by the rate of deciding "Yes, this is the original face which I know from TV/cinema/press" (Figure 2; ORIGINAL: .912 vs. SHIFTED: .537, $t(19)>6.3$, $p<.0001$ ). If exposed to the EXTREME version, this high discriminatory performance was considerably reduced (ORIGINAL: dropped to .656 vs. SHIFTED: raised to .781). Thus, a single exposure to a distorted version of a face resulted in a modification of that face's representation towards the attributes of the extreme face.

Although in Experiment 1 we used a backward mask to delete possible iconic memory traces, we cannot exclude perceptual effects being responsible for the effect found. Therefore, we used an extended delay between exposure and test in Experiment 2.

\section{EXPERIMENT 2}

Experiment 2 was designed to exclude the possibility that the adaptation effect found in Experiment 1 was a simple perceptual/iconic memory or short-term memory aftereffect (Leopold, O'Toole, Vetter, \& Blanz, 2001). Therefore, the exposure and the test were temporally separated by a five minute break. Moreover, a between-subjects design was used with one group to which only EXTREME faces were presented and another group, which was exposed to ORIGINAL faces.

\section{Method}

\section{Subjects}

Forty undergraduate participants from the Freie Universität Berlin were tested individually (30 females, mean age 25.9 years). Twenty randomly selected undergraduates were assigned to the control (ORIGINAL) group (14 females, mean age 25.0), and 20 undergraduates to the experimental (EXTREME) group (16 females, mean age 26.9). All participants were tested individually.

\section{Apparatus and stimuli}

The same material was used as in Experiment 1.

\section{Procedure}

In the exposure phase, the treatment group viewed EXTREME versions for 1-3 seconds, each version presented several times at random places on the screen for 45 seconds altogether. The control group inspected ORIGINAL versions in the exposure phase. In order to familiarize the participants with the faces, both groups were asked to categorize them according to their gender. Note that we used this gender decision task as a cover task to force the participants to not process the face stimuli primarily by their identity or their configural aspects. After a five minute break, the test block began. Both groups were shown ORIGINAL or SHIFTED versions and, as in Experiment 1, the participants decided whether or not each face was veridical.

\section{Results and Discussion}

Similar to the results from the previous experiment, participants who were exposed to EXTREME faces in the exposure phase evaluated SHIFTED versions as veridical more often than participants who were exposed to ORIGINAL faces (EXTREME exposure: .931 vs. ORIGINAL exposure: .643; $t(38)=3.77, p<.001)$, which indicates that the adaptation effect was not only short-termed but was preserved even for five minutes.

Due to the specific design of presenting either ORIGINAL or EXTREME versions in the exposure phase, two alternative explanations for the adaptation effect are possible. On the one hand, a perceptually based mechanism would bias all succeeding images towards the appearance of the inspected face. Such a mechanism would be based on unspecific adaptation, independently of any specific identities. On the other hand, the effect could be based on an identity-based modification with a representational basis. According to this explanation, a face would be stored as a prototype that is updated with every new and deviating 
instance. Therefore, only facial images belonging to the same identity would adapt an existing face prototype. This could be an essential mechanism for a permanent updating process of the representational system. From this perspective, recently processed information would permanently be integrated into the existing memory to optimize succeeding identification processes in order to achieve more accurate and faster recognition in the future. Experiment 3 was designed to test these assumptions by using a variation in the exposure phase with different pictorial versions of one and the same celebrity.

\section{EXPERIMENT 3}

In a final experiment, we asked whether participants tend to select extreme versions as original simply because they bear stronger resemblance to the previously seen pictorial code, rather than their amended stored structural representations of the celebrity's face. The design of this experiment was similar to Experiment 1 with the exception that, in $50 \%$ of cases, a different photograph of the celebrity was used at test.

\section{Method}

\section{Subjects}

Twenty undergraduate participants from the Freie Universität Berlin were tested individually (15 females, mean age 24.0 years).

\section{Apparatus and stimuli}

New faces, each presented by two high quality photographs, were selected. All these faces were highly familiar to German participants (C. Crawford, Madonna, B. Spears, V. Feldbusch, G. Clooney, A. Schwarzenegger, M. Schumacher, T. Gottschalk). A stimulus specific visual mask, composed by an overlay of all stimuli filtered by a Gaussian mask (Carbon, 2003) was used to prevent the persistence of recent iconic impressions (Eriksen, 1980).

\section{Procedure}

The procedure of this experiment was similar to that of Experiment 1. The number of trials was increased to 64 experimental trials (two different base images of test faces per celebrity) plus three preceding blind trials, which were excluded from further analyses. The exposure face was presented for 5 seconds followed by a visual mask based on unspecific face information.

\section{Results and Discussion}

Similar to the findings in Experiment 1, the exposure to distorted faces biased the identification performance towards the EXTREME (cf. Figure 2). This was analyzed by a three-way repeated measurement ANOVA with EXPOSURE (ORIGINAL vs. EXTREME), TEST FACE (ORIGINAL Vs. SHIFTED), and TRANSFER (SAME vs. DIFFERENT base face) as within factors. There were significant main effects of TRANSFER $[F(1,19)=6.89$, $p<.02]$, TEST FACE $[F(1,19)=25.52, p<.0001]$, as well as the two-way interaction between EXPOSURE and TEST FACE $[F(1,19)=34.84, p<.0001]$. Most interestingly, there was a three-way interaction between TRANSFER, EXPOSURE, and TEST FACE $[F(1,19)=$ $8.70, p<.001]$. This three-way interaction indicates that TRANSFER modifies the interaction of EXPOSURE and TEST FACE. The presentation of different pictures of the same person reduced the adaptation effect. This was indicated by the change of interaction from a disordinal to an ordinal one.

However, although less marked, an adaptive mechanism exists for different images of the same person's face, too. This was analyzed by an additional two-way repeated measurement ANOVA with EXPOSURE and TEST FACE as within factors for the different base face trials only. Beside significant main effects for EXPOSURE $[F(1,19)=7.296, p<.02]$ and TEST FACE $[F(1,19)=$ $32.09, p<.0001]$, the analysis revealed a significant interaction between the two factors $[F(1,19)=12.11$, $p<.005]$. Thus, exposure to EXTREME versions again caused a reduction of discrimination performance even when different pictures were used for exposure and test. Interestingly, when using different pictures for exposure and test, only the ability to evaluate the veridicality of SHIFTED versions was diminished $[t(19)=$ $3.87, p<.001]$, but not if ORIGINAL faces had to be evaluated $[t(19)<1$, n.s. $]$.

The use of two different photographs precludes an explanation based on purely pictorial priming or a similarity effect. This is an essential difference to the experiments described above, for which pictorial artifacts cannot be fully excluded, although much care was taken to minimize persisting iconic memory traces. Therefore, Experiment 3 seems to show that the adaptation effect demonstrated here is not only based on a more or less pictorial effect but has a more structural or representational basis.

\section{GENERAL DISCUSSION}

In sum, three experiments demonstrated that perceivers seem to integrate new visual information into a flexible structural or even representational frame. After exposure to apparently alienated versions of famous faces, main attributes of these versions seem to affect face perception of pre-experimental familiar faces to- 
wards the perceptual experience (Experiment 1). This holds even for longer delay between exposure and test (Experiment 2). Moreover, it was shown that the effect is not only a pictorial one, but expands to structurally related face stimuli (Experiment 3). Importantly, future experiments should systematically vary the delay between exposure and test and have to analyze the decay of the effect. Furthermore, it is important to investigate whether non-experimentally seen faces are also influenced by the presentation of experimentally distorted faces and whether the observed effect possibly transfers to categories different from faces.

Only with such future experiments, we can surely propose that the representations of individual faces, for example. face recognition units (FRU, Bruce \& Young, 1986), are adapted. If so, this kind of adaptation effect seems to be a powerful mechanism for (automatically) adapting and refining our represented face prototypes (Hintzman, 1986; Nosofsky, Kruschke, \& McKinley, 1992). With the help of such a flexible adaptation system, humans could be adaptive to, and therefore fit for a constantly changing environment. Finally, such an adaptation mechanism might be the basis of the illusion of having stable representations whilst these representations are in fact being permanently adapted, tuned and optimized (Bruce, 1994).

\section{Acknowledgements}

This research was supported by a grant to Helmut Leder from the Deutsche Forschungsgemeinschaft (DFG, Le1286).

\section{REFERENCES}

Baddeley, A. D. (1998). Human memory. Theory and practice. Needham Heights, MA: Allyn and Bacon.

Benson, P. J., \& Perrett, D. I. (1993). Extracting prototypical facial images from exemplars. Perception, 22, 257-262. www

Bower, G. H., Thompson-Schill, S., \& Tulving, E. (1994). Reducing retroactive interference: An interference analysis. Journal of Experimental Psychology: Learning, Memory, and Cognition, 20, 51-66.

Bruce, V. (1994). Stability from variation: The case of face recognition. The M.D. Vernon memorial lecture. Quarterly Journal of Experimental Psychology: Human Experimental Psychology, 47A, 5-28. Www

Bruce, V., \& Young, A. (1986). Understanding face recognition. British Journal of Psychology, 77, 305-327. Www

Carbon, C. C. (2003). Face processing: Early processing in the recognition of faces (Doctoral thesis). Berlin: DARWIN, Freie Universität Berlin,
URL: http://www.diss.fu-berlin.de/2003/35/.

Carbon, C. C., \& Leder, H. (in press). When feature information comes first! The early processing of inverted faces. Perception.

Cohen, J. D., MacWhinney, B., Flatt, M., \& Provost, J. (1993). PsyScope: A new graphic interactive environment for designing psychology experiments. Behavioral Research Methods, Instruments, and Computers, 25, 257-271.

Coltheart, M. (1980). Iconic memory and visible persistence. Perception \& Psychophysics, 27, 183-228. [ww

Eriksen, C. W. (1980). The use of a visual mask may seriously confound your experiment. Perception and Psychophysics, 28, 89-92. $\overline{w W w}$

Faulkner, T. F., Rhodes, G., Palermo, R., Pellicano, E., \& Ferguson, D. (2002). Recognizing the unreal McCoy: Priming and the modularity of face recognition. Psychonomic Bulletin and Review, 9, 327-334. Www

Hintzman, D. L. (1986). 'Schema abstraction' in a multiple-trace memory model. Psychological Review, 93, 411-428.

Lander, K., \& Chuang, L. (in press). Why are moving faces easier to recognize? Visual Cognition, 12, 429-442.

Leder, H., \& Bruce, V. (2000). When inverted faces are recognized: The role of configural information in face recognition. Quarterly Journal of Experimental Psychology: Human Experimental Psychology, 53A, 513-536. [Www

Leder, H., \& Carbon, C. C. (2004). Part to whole effects and configural processing in faces. Psychology Science, 46, 531-543.

Leder, H., \& Carbon, C. C. (2005). When context hinders. Context superiority versus learn-test-compatibilities in face recognition. Quarterly Journal of Experimental Psychology: Human Experimental Psychology, 58A, 235-250. Www

Leder, H., \& Carbon, C. C. (in press). Face-specific configural processing of relational information. British Journal of Psychology.

Leopold, D. A., O'Toole, A. J., Vetter, T., \& Blanz, V. (2001). Prototype-referenced shape encoding revealed by high-level aftereffects. Nature Neuroscience, 4, 89-94. |www|

Neisser, U. (1967). Cognitive psychology. New York: Appleton-Century-Crofts.

Nosofsky, R. M., Kruschke, J. K., \& McKinley, S. (1992). Combining exemplar-based category representations and connectionist learning rules. Journal of Experimental Psychology: Learning, Memory and Cognition, 18, 211-233. Www

Rhodes, G., Jeffery, L., Watson, T. L., Clifford, C. W. 
G., \& Nakayama, K. (2003). Fitting the mind to the world: Face adaptation and attractiveness aftereffects. Psychological Science, 14, 558-566. |www

Turvey, M. T. (1973). On peripheral and central processes in episodic memory. Psychological Review,
80, 1-52.

Webster, M. A., \& MacLin, O. H. (1999). Figural aftereffects in the perception of faces. Psychonomic Bulletin and Review, 6, 647-653. |www| 